\title{
Three RNA Microenvironments Detected in Fluxional Gene Delivery Polyplex Nano-assemblies
}

\author{
Anisha Shakya, ${ }^{1}$ Hashim M. Al-Hashimi, ${ }^{4 *}$ Mark. M. Banaszak Holl ${ }^{1,2,3^{*}}$ \\ Departments of ${ }^{1}$ Chemistry and ${ }^{2}$ Biomedical Engineering and ${ }^{3}$ Program in Macromolecular Science and \\ Engineering, University of Michigan, Ann Arbor, MI 48190-1055, USA \\ ${ }^{4}$ Department of Biochemistry and Chemistry, Duke University Medical Center, Durham, NC 27710, \\ USA
}




\section{S1. Contents}

This document contains additional information that supports points made in the main text. A full description of the experimental methods and characterizations is given in Section S2. Section S3 shows the size distribution of G5/TAR polyplexes at different $\mathrm{N}: \mathrm{P}$ ratios as measured by dynamic light scattering (DLS). Section S4 shows normalized emission spectra of TAR-FL in presence of a) G5 and b) at different $\mathrm{pH}$ solutions. S5 shows the bulk $\mathrm{pH}$ measurements of polyplex solutions. Section S6 shows effect of a) G5, b) bPEI, and c) 1PEI on free fluorescein spectrum. Section S7 shows normalized emission spectra of TAR-FL in presence of a) bPEI and b) IPEI. Section S8 shows comparison of fluorescence quenching of TAR-FL upon polyplex formation with different POCP. Section S9 contains references cited herein. 


\section{S2. Experimental}

\section{Materials and Chemicals:}

Potassium chloride $(\mathrm{KCl}), \quad 0.1 \quad \mathrm{~N}$ hydrochloric acid $(\mathrm{HCl}), \quad$ tris (hydroxymethyl)-aminomethane, and Triton-X were purchased from Sigma, St. Louis, MO. G5 PAMAM dendrimer was purchased from Dendritech Inc. (Midland, MI) and purified before use using published protocols. ${ }^{1}$ The molecular weight as characterized by GPC was $25680 \mathrm{~g} / \mathrm{mol}$. bPEI was obtained from Sigma Aldrich Corporation with Mw of $\sim 25,000, \mathrm{Mn} \sim 10,000$, and PDI of 2.5. 1PEI was obtained from Polysciences, Inc., with $\mathrm{Mw} \sim 25,000, \mathrm{Mn} \sim 23,750$, and PDI of 1.04. A 29-nt RNA, TAR tagged with fluorescein at the 3' end (TAR-FL), was purchased from Dharmacon (Lafayette, CO) in HPLC purified form with molecular weight $9899.7 \mathrm{~g} / \mathrm{mol}$ and extinction coefficient $289,900 \mathrm{~L} / \mathrm{mol} \mathrm{cm}$ as specified by the vendor.

\section{Fluorescence Spectroscopy:}

All oligos were annealed by heating to $95^{\circ} \mathrm{C}$ for $5 \mathrm{~min}$ and then cooled on ice for an hour prior to use. The oligos were diluted in $\mathrm{pH} 7.54$ fluorescence assay buffer (50 $\mathrm{mM}$ Tris- $\mathrm{HCl}, 50 \mathrm{mM} \mathrm{KCl}, 0.01 \%$ Trition-X). Polymers were dissolved in the assay buffer and serially diluted to obtain aliquots of concentration from $2 \mathrm{nM}$ to $2000 \mathrm{nM}$. $100 \mathrm{nM}$ oligo was incubated for $30 \mathrm{~min}$ in 1:1 volume with varying concentrations of the dendrimer in a 384 well plate such that the final oligo concentration in each well was $50 \mathrm{nM}$ and varying concentration ratios. Fluorescence emission at wavelengths $500-600 \mathrm{~nm}$ with stepwidth of $1 \mathrm{~nm}$ using $472 \mathrm{~nm}$ excitation wavelength and $16 \mathrm{~nm}$ bandwidth of excitation. Measurements were done in triplicates using a CLARIOStar Omega plate reader (BMG Labtech) at room temperature.

\section{Bulk pH measurements:}

Polyplex solutions were prepared in the same buffer conditions and concentrations as prepared for fluorescence experiments, but in higher volumes in order to facilitate $\mathrm{pH}$ measurement using a $\mathrm{pH}$ probe. The probe was dipped into the polyplex solution and allowed to equilibrate until the $\mathrm{pH}$ reading was stable. Both calibration and measurements were performed at room temperature.

Hydrodynamic diameter characterization of polyplexes using DLS:

G5-TAR polyplexes prepared under the same conditions as in fluorescence experiments described above. The hydrodynamic diameter was measured at room temperature using Malvern Zetasizer Nano ZS (Worchestershire, United Kingdom). Three rounds of measurements were performed for each sample, with the diameter obtained in each round being an average of at least 11 measurements with duration of $20 \mathrm{~s}$. The dispersant viscosity and refractive index were assumed to be that of water (cP 0.8872 and 1.33 , respectively). 


\section{S3. Size characterization of G5/TAR polyplexes using DLS}
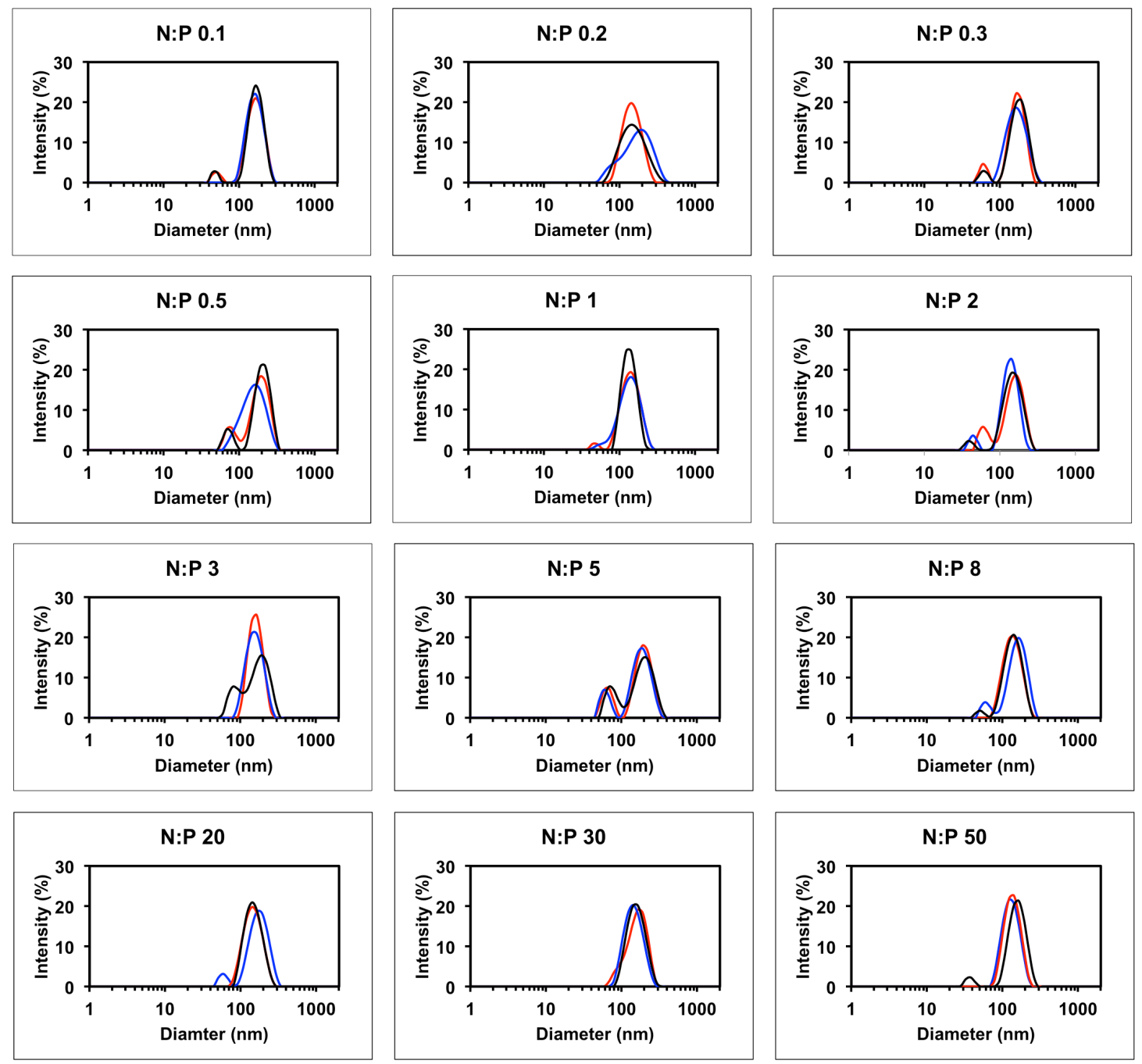

Figure S3. Hydrodynamic diameter of G5/TAR polyplexes prepared under the conditions of fluorescence measurements at different N:P ratios measured using DLS. Triplicate measurements are reported in each graph. 
S4. Normalized emission spectra of TAR-FL

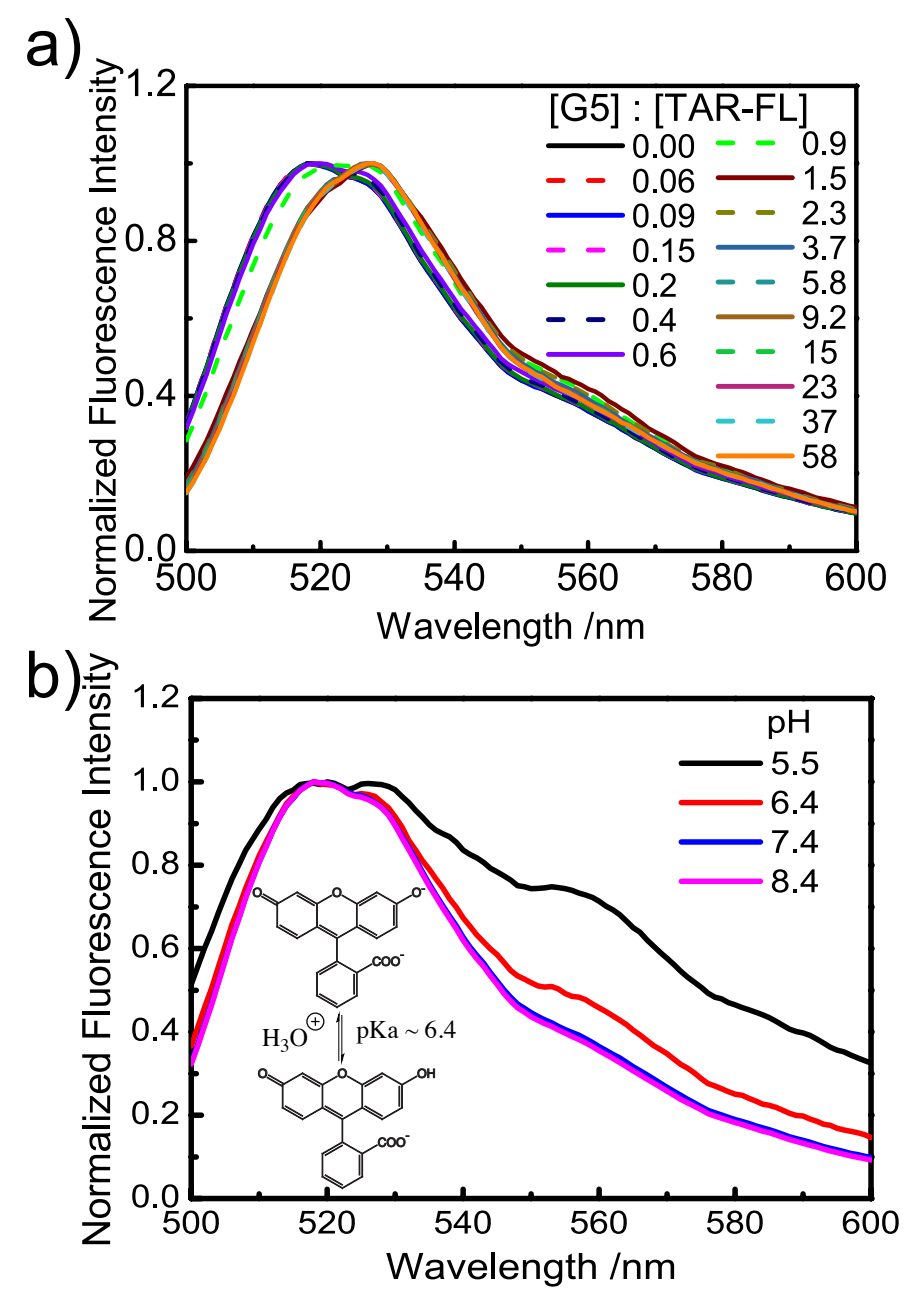

Figure S4. a) Normalized steady state emission spectra (excitation wavelength, $\lambda_{\mathrm{ex}}=$ $472 \mathrm{~nm})$ of TAR-FL at various G5:TAR-FL concentration $(\mu \mathrm{g} / \mathrm{mL})$ ratios. Solutions were buffered at a $\mathrm{pH}$ of 7.54 using tris-HCL. b) Normalized emission spectra of TAR-FL at different buffer (tris- $\mathrm{HCl}) \mathrm{pH}\left(\lambda_{\mathrm{ex}}=472 \mathrm{~nm}\right)$. Also shown is the equilibrium between two fluorescent deprotonated forms of fluorescein that are present in aqueous solutions in the $\mathrm{pH}$ range $5.5-8.4$; the monoanion ( $\mathrm{FL}^{-}$) and dianion $\left(\mathrm{FL}^{2-}\right.$ ) with $\mathrm{pKa}$ of transition $\sim 6.4 .^{2,3}$ 


\section{S5. Bulk solution pH for G5/TAR-FL polyplex solutions}

Table S5. Bulk solution pH of G5/TAR-FL polyplex solutions

\begin{tabular}{|r|r|c|}
\hline $\mathrm{N}: \mathrm{P}$ & $\mu \mathrm{g} / \mathrm{mL}$ ratio & $\mathrm{pH}$ \\
\hline 0.00 & 0.00 & 7.54 \\
\hline 0.07 & 0.06 & 7.54 \\
\hline 0.11 & 0.09 & 7.53 \\
\hline 0.18 & 0.15 & 7.54 \\
\hline 0.28 & 0.23 & 7.54 \\
\hline 0.45 & 0.37 & 7.54 \\
\hline 0.71 & 0.58 & 7.54 \\
\hline 1.13 & 0.92 & 7.54 \\
\hline 1.79 & 1.46 & 7.53 \\
\hline 2.83 & 2.32 & 7.54 \\
\hline 4.49 & 3.67 & 7.53 \\
\hline 7.11 & 5.82 & 7.54 \\
\hline 11.27 & 9.22 & 7.53 \\
\hline 17.86 & 14.62 & 7.54 \\
\hline 28.31 & 23.16 & 7.53 \\
\hline 44.87 & 36.71 & 7.54 \\
\hline
\end{tabular}




\section{S6. Effect of POCPs on free fluorescein emission spectrum}

a)

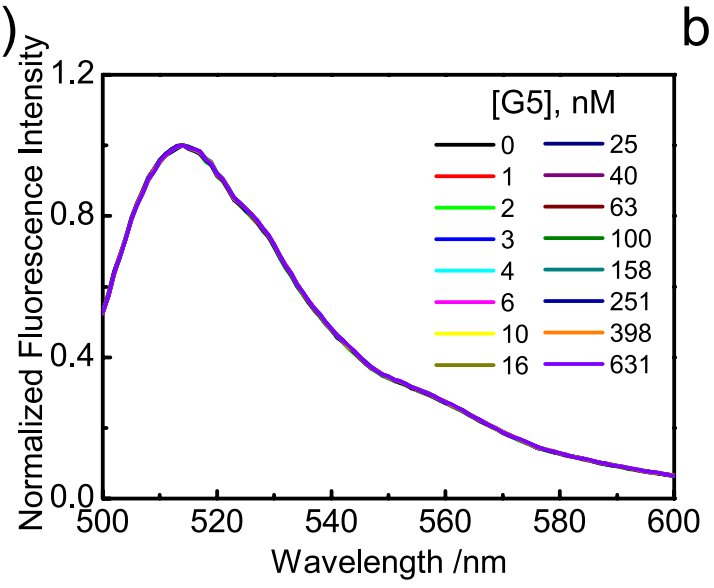

b)

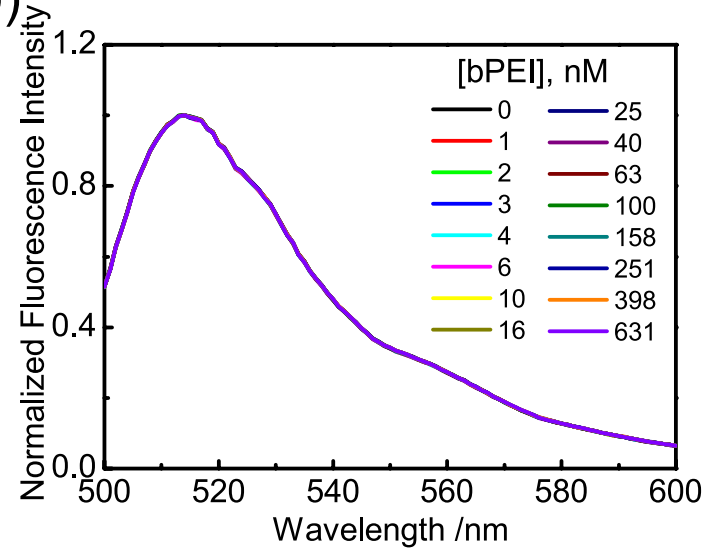

c)

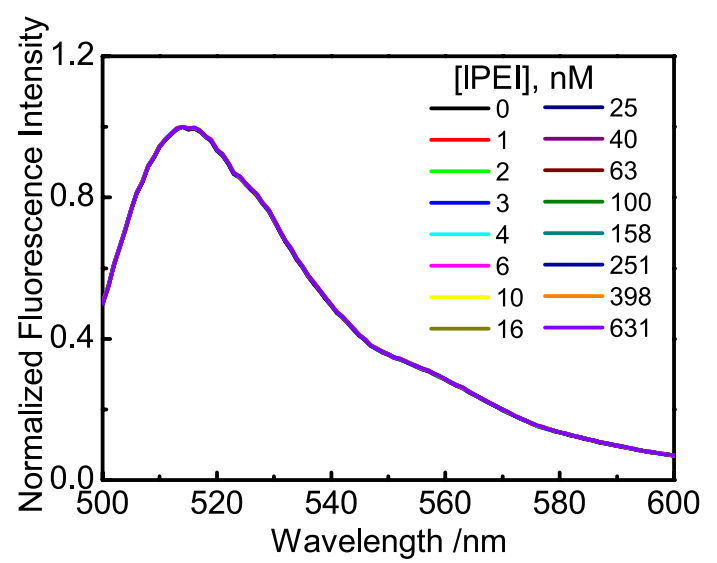

Figure S6. Effect of a) G5, b) bPEI, and c) 1PEI on free fluorescein spectrum. The concentration of fluorescein was $50 \mathrm{nM}$ and the concentrations of POCP used were same as in presence of polyplex formation experiments. All the spectra at different POCP concentrations overlap with each other due to insignificant changes in the free fluorescein spectrum. 
S7. Fluorescein emission spectra of TAR-FL upon polyplex formation with bPEI and IPEI
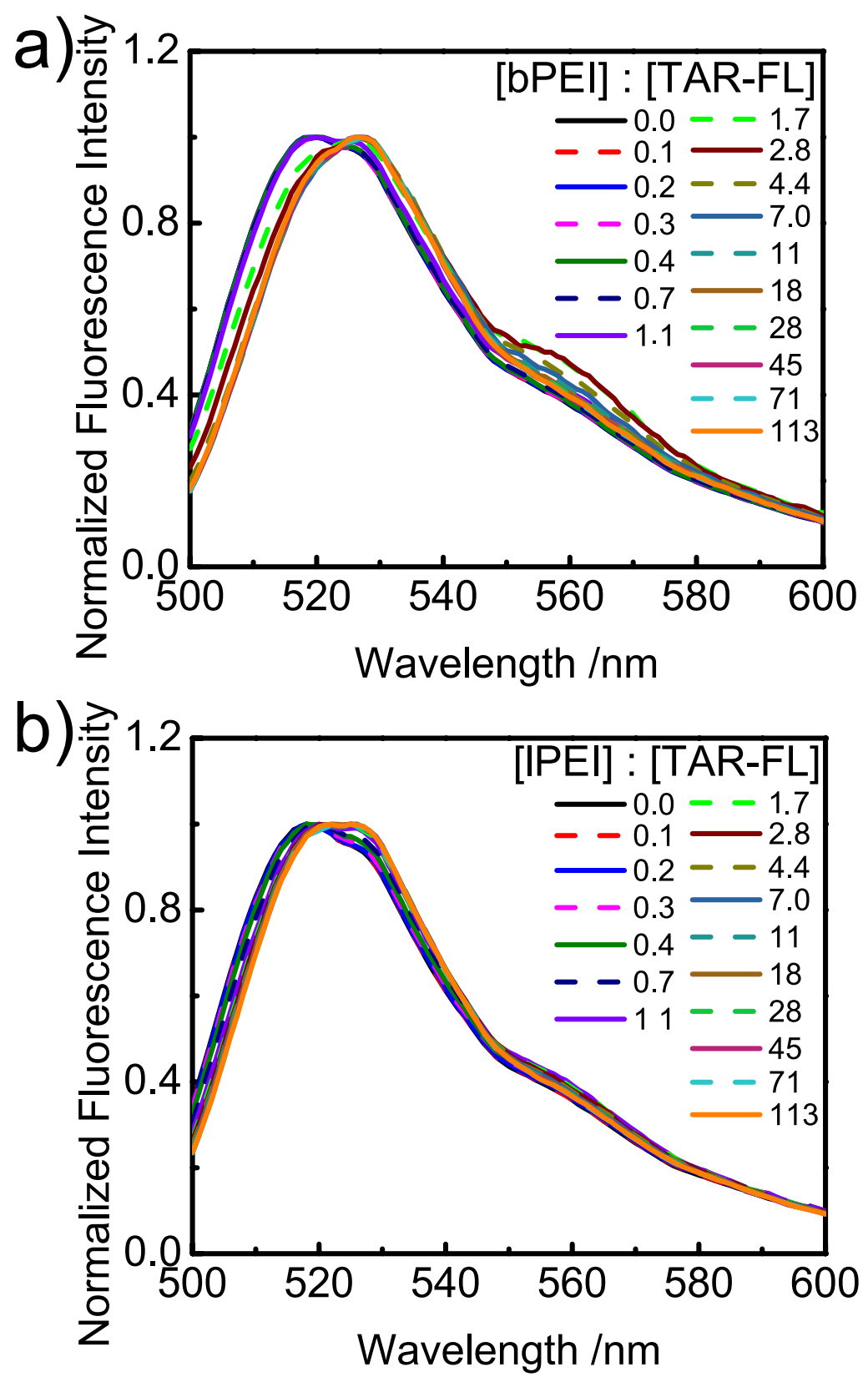

Figure S7. Normalized emission spectra $\left(\lambda_{\mathrm{ex}}=472 \mathrm{~nm}\right)$ of a) bPEI/TAR-FL polyplexes at different bPEI to TAR-FL concentration $(\mu \mathrm{g} / \mathrm{mL})$ ratios b) IPEI/TAR-FL polyplexes at different IPEI to TAR-FL concentration $(\mu \mathrm{g} / \mathrm{mL})$ ratios. 
S8. Comparison of fluorescence quenching of different POCPs

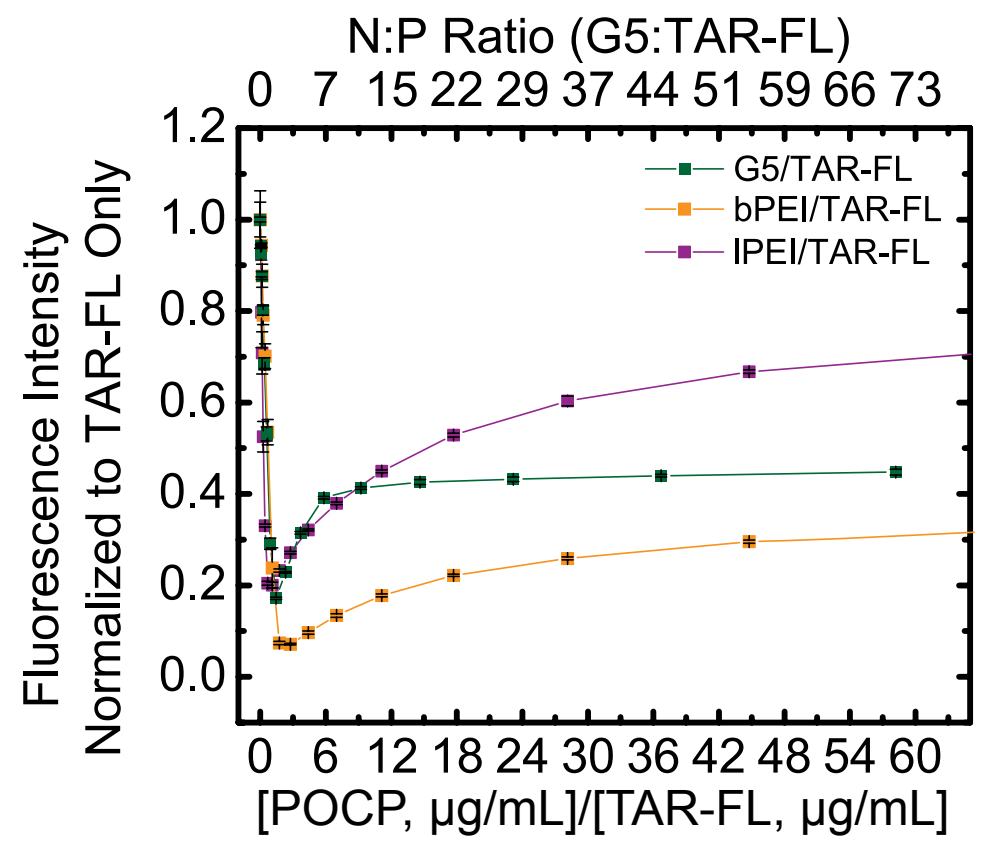

Figure S8. Comparison of fluorescence quenching of TAR-FL upon polyplex formation with different POCP as a function of POCP to TAR-FL concentration ratios.

\section{S9. References:}

(1) Mullen, D. G.; Desai, A.; van Dongen, M. A.; Barash, M.; Baker, J. R., Jr.; Holl, M. M. B. Best Practices for Purification and Characterization of PAMAM Dendrimer. Macromolecules 2012, 45, 5316-5320.

(2) Sjoback, R.; Nygren, J.; Kubista, M. Absorption and Fluorescence Properties of Fluorescein. Spectrochim. Acta A 1995, 51, L7-L21.

(3) Yguerabide, J.; Talavera, E.; Alvarez, J. M.; Quintero, B. Steady-State Fluorescence Method for Evaluating Excited-State Proton Reactions - Application to Fluorescein. Photochemistry and Photobiology 1994, 60, 435-441. 